\title{
Conception and Validation of Smart Building Energy Management System BEMS Using the Discrete Event System Specification DEVS
}

\author{
Abdelfettah Maatoug, and Ghalem Belalem
}

\begin{abstract}
The improvement of the energetic behavior of buildings has turned into a major issue due to the high level of energy consumption. In this context, the building is represented as a dynamical system and a system of data acquisition is developed, which allows the measurement of environmental and energetic parameters, so as to describe the behavior of the building interacting with its direct environment. Research related to energy management can be divided into two categories: predictive control (anticipative) and adaptive control (reactive). A new building energy management system (BEMS) which is the chosen system to validate, treats a long-term anticipative control and introduces a reactive control that adds another level of intelligence to the BEMS.
\end{abstract}

The main goal of this paper is to propose a model using the formalism DEVS to describe and simulate the BEMS. Our motivation is explained by the fact that DEVS is a tool for modeling of discrete event systems and it divides the overall system into subsystems in order to facilitate the achievement which is consistent with the characteristics of multilayer architecture of the chosen system.

Index terms: Smart building, Building energy Management system BEMS, Multilayer architecture, Modeling and simulation DEVS

\section{INTRODUCTION}

Energy plays a vital role in the economic and social development and improving the quality of life. Much of the world's energy, however, is produced and consumed in a way that would not be viable in the long term if technology were to remain constant and if overall quantities were to increase substantially. Building is an important energy node and an ideal support to develop and analyze the effects of an Energy Management System (EMS). Because of its potential impact, such a management of global energy demand is important.

To study the performance of EMS, it was necessary to build and configure specific patterns of domestic loads, energy sources, introduce and formalize the subscription constraints,

Manuscript received March 15, 2014; revised June 4, 2014.

This work has been partially supported by Computer Science Laboratory of Oran (LIO) University of Oran, Algeria, in 2014.

Authors are with National Superior School of Computer Science (ESI), Algiers, Algeria, and University of Oran, Faculty of Exact and Applied Sciences, Oran, Algeria. E-mails: a_maatoug@esi.dz, ghalem1dz@univ-oran.dz. variable cost of purchase and resale of energy and finally, taking into account the preferences of the occupants. We study indeed a BEMS that used to adjust consumption to available energy resources while maximizing the comfort of occupants.

Several research projects have helped to develop tells systems. All this research can be divided into two categories: predictive control (anticipative) and adaptive control (reactive). Most recent developments in BEMS use the concept of predictive control. This control uses a model with measured data to predict the strategy of the optimal control of implementation. The predictive control is used, an error of forecasting short-term (10-20 min) or long term (one day) for the control of temperature or humidity.

D.L. Ha (2006) proposed a management system with anticipative and reactive management of building control, as is the case of the BEMS to validate in this paper, which treats an anticipative long-term (one day) control and introduced the reactive control that adds another level of intelligence of the BEMS. The architecture and the functioning of the chosen management system are detailed in this paper.

Modeling and simulation are essential tools to analyze the behavior of dynamic systems. Several methods have been proposed to improve the process of analyzing the behavior of these systems. These proposals attempt to achieve more realistic models, relatively simple and highly flexible. This work is focused on modeling and simulation of a BEMS using the characteristics of the formalism DEVS for the first time. Our motivation is explained by the fact that DEVS is a tool for modeling of discrete event systems and it divides the overall system into subsystems in order to facilitate the achievement which is consistent with the characteristics of multilayer architecture of the chosen system.

This paper is organized as follows: In section 2 we quote related works for BEMS. In the section 3 we present the multilayer architecture of the chosen system. In section 4 we introduce the basic concepts of DEVS. In section 5 we propose a multilevel DEVS modeling for the BEMS and we discuss the results of the simulation. Finally we conclude our work in section 6 and we give our future prospects.

\section{RELATED WORKS}

Several researches led to the development of energy management systems in buildings: 


\section{A. Energy Managers}

Before giving the architecture and the mechanism of the selected BEMS, we will quote some energy managers:

In [1] and [2], Wacks began to introduce the control of the energy demand in the home using a domestic system. The concept of EMS in buildings is shown in [3]. This system consists of a set of devices using micro controllers which having communication capabilities via standard protocols.

In [4], Andersen and Klaus tried to combine the traditional method for estimating the physical parameters of the building with the identification of stochastic models.

In [5], a distributed approach of the load of network based on the paradigm of Multi-Agent Systems at a communication level is presented.

In [6], the features and capabilities of BEMS are summarized in three categories:

- The basic functions are general functions fitted to the most current systems;

- The intermediate functions are functions in course that can be implemented in the near future;

- Advanced features that are under development.

In [7], a Multi-Agent System for the control of energy in the tertiary building is presented. The purpose of this system is to provide three services, light, heating and ventilation and also to minimize energy consumption in the offices.

Recently, in [8], Kampf and Robinson used a type of simplified models to analyze the transmission of energy flows in multi zone building. In [9], a portrait of future building is presented with technological solutions that integrate systems of energy production to the building from renewable energy sources.

Management of energy flows in the building aims so to meet several criteria that can be divided into three main categories:

- Criteria for user comfort;

- Economic and financial criteria which correspond to the costs of investment and operation;

- Environmental criteria that correspond to the reduction of pollution and compliance with environmental constraints.

In [10], Donsez and al. are interested in comfort criteria of the occupant. They offer a platform oriented service to facilitate the development of a domestic system. However, this system is only interested in the appearance of comfort without taking into consideration the fact that the capacity of energy sources are limited in terms of production.

In [11], Dodier and Henze have developed a learning mechanism that provides an adaptive strategy of control/command taking into account the consumption and the prediction of the energy produced by photovoltaic panels. The result showed that this strategy has not significantly improved the management of energy production.

In [12], an advanced system of the management energy in the building is offered. The objective of this system is to

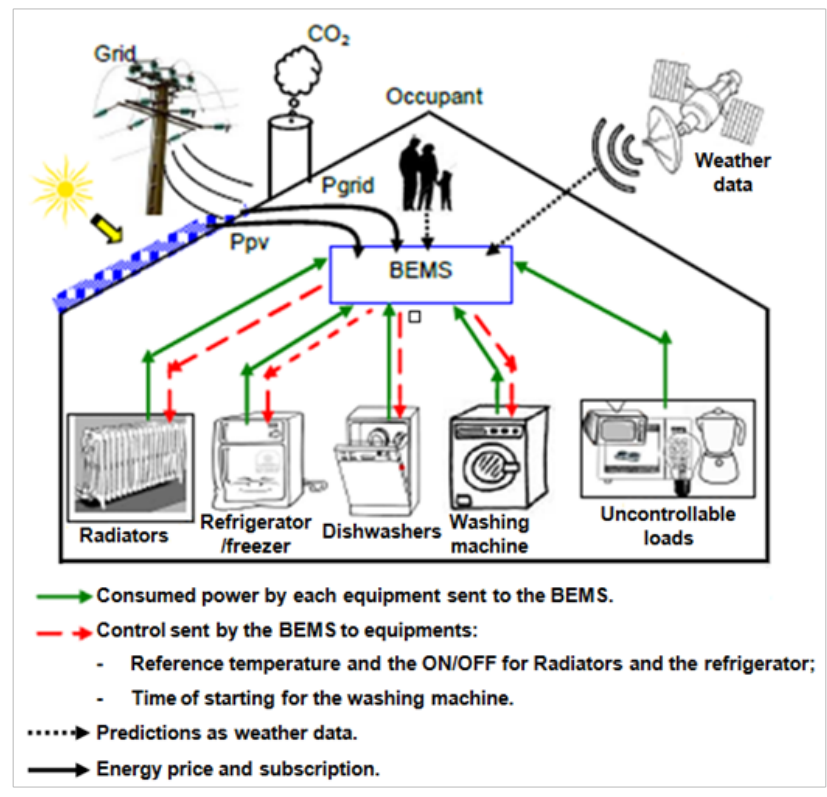

Fig. 1. Energy management in building

freedom degrees by the user and related operation equipments. Flexibilities are able to modify the operation of equipments, for example: shift or interruption of operation. This is done by providing domestic equipments and communication skills using the appropriate algorithms.

All this research can be divided into two categories: predictive control (anticipative) and adaptive control (reactive: real-time). Most recent developments in BEMSs use the concept of predictive control. This control uses a model with measured data in order to predict the strategy of the optimal control of implementation [13]. The predictive control is used, an error of forecasting short-term (10-20 min) or long term (one day) to control the temperature or the humidity.

These projects correspond to different cases of applications, but they have a common objective to the establishment of an energy management system in a building in order to optimize the use of energy (Fig. 1) [14].

In [15], a Multi-agent Distributed System which defines two levels of BEMS control at different horizons of time is proposed: the Reactive mechanism and the Anticipative mechanism. In [16], a management system is offered in order to maximize the user comfort as well as the economic and the environmental costs, with anticipative and reactive control of building management, as is the case of the BEMS to validate, that treats a anticipative long-term control (one day) and introduces the reactive control that adds another level of intelligence to the BEMS.

\section{ARChITECTURE OF THE SYSTEM}

The design of multi-layer optimization system can meet the three requirements own of BEMS: Comfort, Economic, and Environment.

Several levels of modeling are used in this architecture. They correspond to several layers of command to divide the overall problem into sub-problems to be more responsive to uncertainties. Between these layers, a flow of information is 
used to exchange instructions and emergency messages. These levels of management correspond to prioritized algorithms for solving according to different time scales.

An optimization solution is calculated at the highest level, that is to say, with the longer sampling period, taking into the relatively inaccurate predictions. The solution obtained is then refined in a lower level of management that fits the solution already calculated taking into account more detailed information for a smaller sampling period. The solution should converge towards real instructions to equipments in the building, or towards advices to occupants.

This architecture of multi-levels management must address to the major problem in the management of energy flows: the prevailing of uncertainties in the model of behavior of services in the external conditions and especially in the behavior of forecasts and demands of people.

Management architecture in three layers proposed by D. L. Ha [16]: an anticipative layer, a reactive layer and a local layer is shown in Fig. 2.

The system treats an anticipative long-term (one day) control and introduced the reactive control that adds another level of intelligence of the BEMS.

This architecture consists of several control layers. They are distinguished in depending of the optimization horizon and different sampling periods:

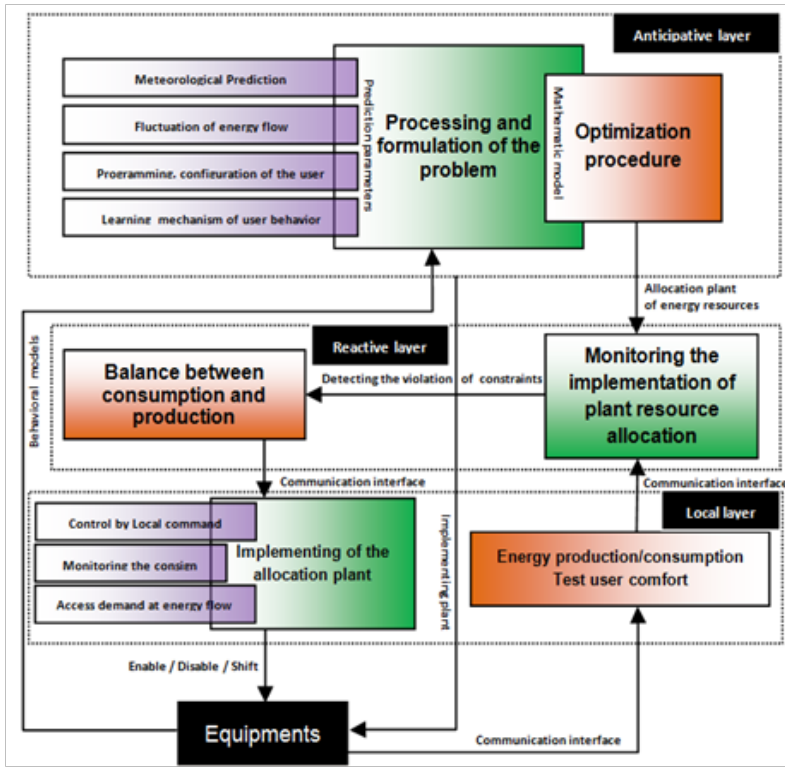

Fig. 2. Multi-layers architecture of piloting for energy management in the habitat [16]

- Anticipative layer is to plan consumption and production of energy when some events are planned hours or a day in advance. The horizon of this control layer is typically one day and the time of sampling is of the order of one hour.

- Reactive layer is a complement to the anticipative layer. It helps anticipative layer to carry out the plan of allocation of energy resources taking into account energy constraints and comfort of occupants. The reactive layer has a short time horizon of the order of one minute but a much faster response time than that anticipative layer to absorb disturbances.
- Local control layer is linked to the control system and control equipment. Its role is to follow the guidelines from the reactive layer [16].

In this work, we focus on the first two layers of control because the local layer is a matter for equipment manufacturers. Our goal is not only to model these control layers but also to validate their operation and the energy management mechanism by varying predicted scenarios (from the BEMS) and real data (simulation values from the simulation model of building).

\section{THE DEVS FORMALISM}

In this section we introduce the basic concepts of the formalism DEVS.

B.P. Zeigler defined in [17], a formal specification of discrete event models. This formalism was introduced as an abstract universal formalism independent of the implementation. The DEVS formalism is based on the definition of two types of models: Atomic models and Coupled models.

Atomic models used to represent the basic behavior of the system. Coupled models are defined by a set of sub atomic models and/or coupled models to represent the internal structure of the system through coupling between models.

\section{A. Formal specification of a DEVS atomic model}

The atomic model provides an autonomous description of behavior system, defined by states and input/output functions and internal transitions of the model. The evolution of the model is done by state change according to external stimuli (via an input) or internal (via a transition function). These changed states are intended to determine the behavioral response of the system to these stimuli (Fig. 3).

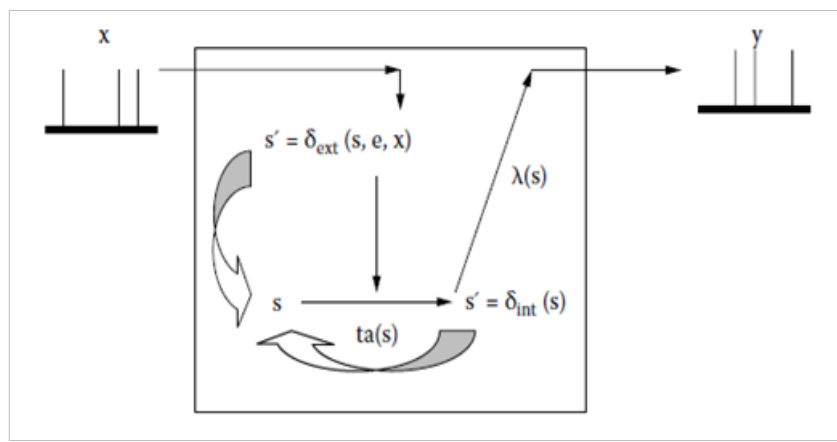

Fig. 3. Description of an atomic model DEVS

An atomic model AM is defined by [18]:

$$
A M=\left(X, Y, S, \delta_{\text {ext }}, \delta_{\text {int }}, \lambda, t_{a}\right)
$$

$\mathbf{X}$ : set of external events (input).

$\mathbf{Y}$ : set of output events.

$\mathbf{S}$ : set of states.

$\delta_{\text {int }}: S \rightarrow S$ : internal transition function caused by the occurrence of internal events.

$\delta_{\text {ext }}: Q \times S \rightarrow S$ : external transition function caused by the occurrence of external events, $\mathrm{Q}=\left\{(\mathrm{s}, \mathrm{e}) \mid \mathrm{s} \in \mathrm{S} .0 \leq \mathrm{e} \leq \mathrm{t}_{\mathrm{a}}(\mathrm{s})\right\}$ : 
Total states and e describes the elapsed time since the system made a transition to the current state $\mathrm{s}$.

$\lambda$ : output function.

$\mathbf{t}_{\mathbf{a}}$ : function of lifetime of state, represents the maximum time during which the model remains in a state $s \in \mathrm{S}$.

\section{B. Formal specification of a DEVS coupled model}

To more describe a complex system we interconnect several atomic models to form a coupled model. This new model can be used as a basic model in a higher level description; this is the hierarchical aspect of the formalism (Fig. 4).

A coupled model CM is defined by [18]:

$$
C M=\left(X, Y, D,\left\{M_{d} / d \in D\right\}, E I C, E O C, I C\right)
$$

$\mathbf{X}$ : set of possible inputs of the coupled model.

Y: set of possible outputs of the coupled model.

D: set of names associated to the model components.

$\mathbf{M}_{\mathbf{d}}$ : set of the coupled model components, these components are either atomic or coupled DEVS model.

EIC: set of External Input Coupling.

EOC: set of External Output Coupling.

IC: set of Internal Couplings.

Coupled model consists of sub-models that can be atomic or coupled and has the following three relations coupling:

- A relationship of internal coupling for coupling between the ports of sub-models that make up the coupled model.

- A coupling relation of external inputs for the coupling between the input ports of the coupled model and the input ports of the sub-models.

- A coupling relation of external outputs for the coupling between the output ports of the coupled model and output ports of the sub-models.

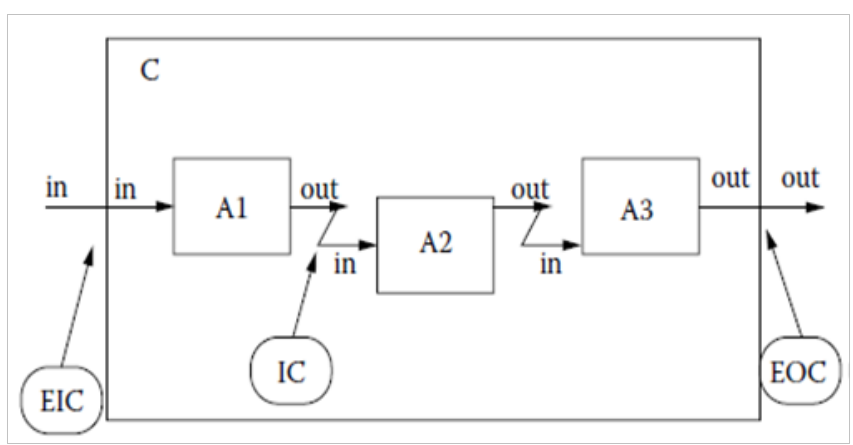

Fig. 4. Description of a coupled model DEVS

\section{CONCEPTION AND VALIDATION OF THE BEMS}

\section{A. Modeling of the BEMS in DEVS}

We have designed the model above for building respecting the multilevel architecture which has been described previously.

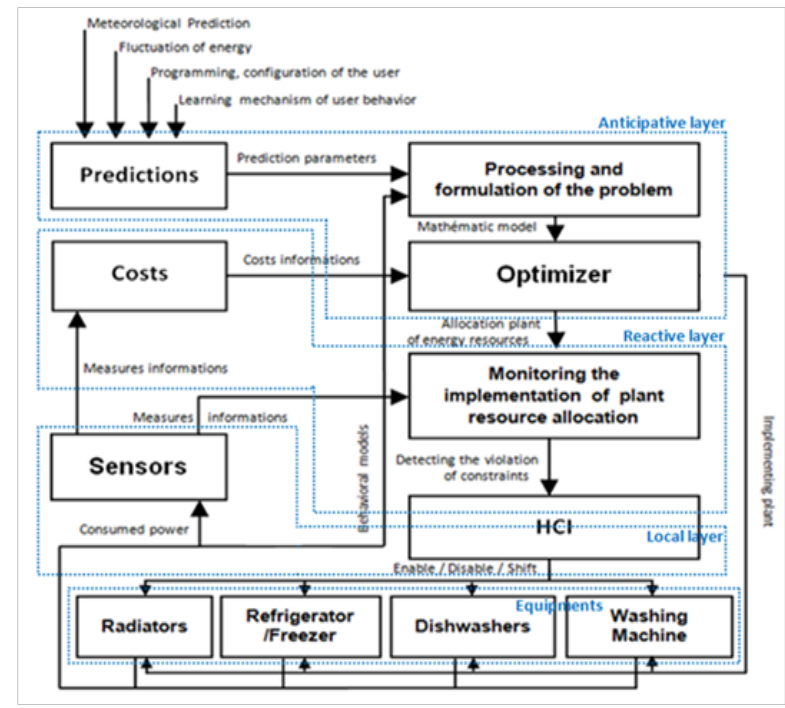

Fig. 5. Multilevel model for the energy management in building

\section{A.1 The formalization of the proposed model in DEVS}

The proposed model divides the BEMS system into eight subsystems: Predictions, Processing and formulation of a problem, Costs, Optimizer, Monitoring the implementation of plant resource allocation, HCI, Sensors, Equipments.

We give to the BEMS the following structure of the DEVS coupled model:

$$
\begin{gathered}
\text { BEMS }=<X, Y, D,\left\{M_{d} / d \in D\right\}, \text { EIC, EOC, IC }> \\
X=\left\{(p, v) / p \in \text { IPorts, } v \in X_{p}\right\} \quad \text { Inputs values } \\
Y=\left\{(p, v) / p \in \text { OPorts, } v \in Y_{p}\right\} \quad \text { Outputs values }
\end{gathered}
$$

D: set of names of the atomic or coupled models that compose the BEMS: $D=\{$ Predictions, Processing and formulation of a problem, Costs, Optimizer, Monitoring the implementation of plant resource allocation, HCI, Sensors, Equipments \}

$\mathbf{M}_{\mathbf{D}}$ : set of atomic or coupled the models that compose the BEMS:

$\mathbf{M}_{\text {Predictions }}$ is the model of Predictions subsystem.

$\mathbf{M}_{\text {costs }}$ is the model of costs subsystem....and so on.

$E I C=\left\{((B E M S, a),(d, b)) / a \in\right.$ IPorts $_{s}, b \in$ IPorts $\left._{d}\right\}$

EOC $=\left\{((d, b),(B E M S, a)) / a \in\right.$ OPorts $_{\text {BEMS }}, b \in$ OPorts $\left._{d}\right\}$ $I C=\left\{((i, a),(j, b)) / i, j \in D, i \neq j, a \in\right.$ OPorts $_{i}, b \in$ IPorts $\left._{j}\right\}$

EIC defines ports of BEMS model that are connected to ports of components models that receive external events. EOC defines ports of BEMS model that are connected to ports of components models that emit events. 


\section{A.2 Representation of the model using the JDEVS tool}

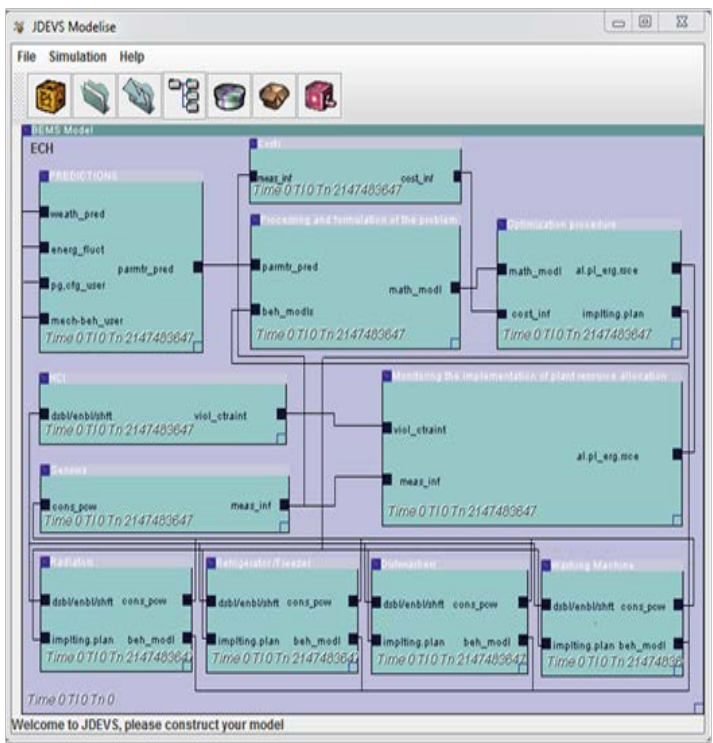

Fig. 6. Representation of the BEMS model with JDEVS

Fig. 6 shows the proposed BEMS model modeled on JDEVS environment. This model consists of several atomic models. The Anticipative layer contains Predictions, processing and formulation of the problem, optimization procedure atomic models, the Reactive layer contains Monitoring the implementation of plant resource allocation, HCI, Costs, the Local layer contains HCI, Sensors, and Equipments contains Radiators, Refrigerator/ Freezer, Dishwashers and Washing machine.

In general, the main objective of validation is to check if a system works as expected, in different conditions and scenarios of simulation. The validation aims to make the model useful in the sense that it responds to good problem.

\section{B. Simulation of a thermal model: a case of application}

There are three types of services: Permanent services (Heating, Fridge, and Freezer), Temporary services (Washing machine and Dishwasher) and Provider services (Grid and PV source) [19]. Table I summarizes the variable measurements and checks exchanged for these services in both files measurements properties and controls properties:

TABLE I.

EXCHANGED MEASURES AND CONTROLS

\begin{tabular}{|c|c|c|}
\hline & Measurements.Properties & Controls.Properties \\
\hline \multirow{2}{*}{$\begin{array}{l}\text { Permanent } \\
\text { services }\end{array}$} & $\begin{array}{l}0=3 . p o w e r[w a t t] \\
1=3 . p o w e r[w a t t]\end{array}$ & $\begin{array}{l}0=3 . \text { temperature [Kelvin }] \\
1=3 . \text { enable }\end{array}$ \\
\hline & \multicolumn{2}{|c|}{3 is an ex of the identifying of a same permanent service } \\
\hline \multirow{2}{*}{$\begin{array}{l}\text { Temporary } \\
\text { services }\end{array}$} & 2=6.power[watt] & $\begin{array}{l}2=6 . \text { relative_starting_time[sd] } \\
3=6 . \text { enable }\end{array}$ \\
\hline & \multicolumn{2}{|c|}{6 is an ex of the identifying of a same temporary service } \\
\hline \multirow{2}{*}{$\begin{array}{l}\text { Provider } \\
\text { services }\end{array}$} & $8=1$.power[watt] & $* * * * *$ \\
\hline & \multicolumn{2}{|c|}{$\begin{array}{l}\text { The BEMS does not control this two last services, it just need } \\
\text { their consumption informations }\end{array}$} \\
\hline
\end{tabular}

Cases of applications include:
PV sources, RE, and the following loads:

TABLE II.

AVAILABLE LOADS IN THE BUILDING

\begin{tabular}{|l|l|l|l|l|}
\hline \multicolumn{1}{|c|}{ Equipments } & \multicolumn{1}{|c|}{$\begin{array}{c}\text { Power } \\
(W)\end{array}$} & Number & Command & $\begin{array}{c}\text { Interrup- } \\
\text { tible }\end{array}$ \\
\hline Radiator & 1500 & 1 & $\mathrm{~T}_{\text {cgn/ON_OFF }}^{\circ}$ & Yes \\
\hline Refrigerator & 150 & 1 & $\mathrm{~T}_{\text {cgn/ON_OFF }}^{\circ}$ & Yes \\
\hline Freezer & 200 & 1 & $\mathrm{~T}_{\text {cgn/ON_OFF }}^{\circ}$ & Yes \\
\hline Washing machine & 2000 & 1 & $\mathrm{t}_{\text {of START }}$ & Not \\
\hline Dishwasher & 2200 & 1 & $\mathrm{t}_{\text {of START }}$ & Not \\
\hline $\begin{array}{l}\text { uncontrollable } \\
\text { loads }\end{array}$ & Vary & globalized & Nothing & Not \\
\hline
\end{tabular}

We are interested in the energy behavior of Refrigerator without and with using the BEMS to test the operation of the system.

\section{B.1 The Refrigerator sub-model}

The Refrigerator is a thermal service as it is regulated as follows: it is either in the stop $(P=0 \mathrm{~W})$ or in the maximum power $\left(P=P_{\max }\right)$. So $\mathbf{t}_{\text {cold }}$ is the period of operation at $\mathbf{P}_{\max }$ and $\mathbf{t}_{\text {hot }}$ the period of stop.

The model is [19] :

\section{B.1.1 At the period «t $\mathbf{t}_{\text {cold }}$ »}

$d\left(T_{\text {Ref }}-T_{\text {out }}\right) / d t=-1 / \tau\left(T_{\text {Ref }}-T_{\text {out }}\right)+k / \tau\left(P_{\text {max }}\right)$

$\mathbf{T}_{\text {Ref: }}$ inside temperature (Refrigerator temperature) [ $\left.{ }^{\circ} \mathrm{K}\right]$ $\mathbf{T}_{\text {out }}$ : outside temperature (Room temperature) [ $\left.{ }^{\circ} \mathrm{K}\right]$ k: A factor of cold generation for Refrigerator.

$\tau$ : Time constant of Refrigerator [sd] such that $\tau=\mathrm{R} \times \mathrm{C}$ with "R" in [KW] is the thermal resistance and " $\mathrm{C}$ " in $[\mathrm{J} \mathrm{K}]$ is the calorific capacity of the Refrigerator.

By integrating (4) we get:

$T_{\text {in }}-\Delta t-T_{\text {out }}=e^{-t_{\text {cold } / \tau}}\left(T_{\text {in }}+\Delta t-T_{\text {out }}\right)+k\left(1-e^{-t \text { cold } / \tau}\right) P_{\max }$

$\mathbf{T}_{\mathbf{i n}}$ : Rerfrigerator temperature of consign.

$\Delta \mathbf{T}$ : Variation of maximum temperature allowed for the command (here $=1{ }^{\circ} \mathrm{K}$ ).

B.1.2 At the period «t $\mathbf{h o t}_{\text {» }}$

$d\left(T_{\text {Ref }}-T_{\text {out }}\right) / d t=-1 / \tau\left(T_{\text {Ref }}-T_{\text {out }}\right)$

By integrating (6) we get :

$T_{\text {in }}+\Delta t-T_{\text {out }}=e^{-t_{\text {hot }} / \tau}\left(T_{\text {in }}-\Delta t-T_{\text {out }}\right)$

The energy management system calculates the temperature of consign and imposes it on the system.

The model provided the periods of operation $t_{\text {hot }}$ and $t_{\text {cold }}$ as a function of the setpoint temperature of the room in which the Refrigerator is located, and the intrinsic parameters of the Refrigerator, and power consumption based on costs.

B.2 Formalization of the Refrigerator sub-model in DEVS and Simulation using the JDEVS tool 


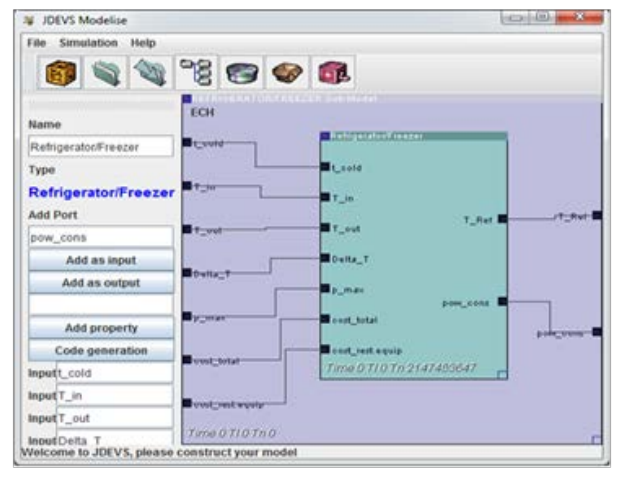

Fig. 7. Representation of the Refrigerator sub-model in JDEVS

We have formalized the Refrigerator subsystem by the structure of an atomic model DEVS as follows :

Refrigerator $=\left(X, Y, S, \delta_{\text {ext }}, \delta_{i n t}, \lambda, t_{a}\right)$

$X: t_{\text {cold }}, T_{\text {in }}, T_{\text {out }}, \Delta t, P_{\max }$, cost $t_{\text {total }}$, cost $_{\text {rest.equip }}$ (inputs).

S: transition states "Off", "On".

Y: $T_{\text {Ref }}$ Refrigerator temperature and pow cons $_{\text {s }}$ the power consumed by the Refrigerator (outputs).

$\delta_{\text {ext }}$ : Calculates the temperature supplied and the power consumed by the Refrigerator by the following formula :

$\left\{\begin{array}{l}T_{\text {Ref }}=e^{-t c o l d / \tau}\left(T_{\text {in }}+\Delta t-T_{\text {out }}\right)+k\left(1-e^{-t c o l d / \tau}\right) P_{\text {max }} \\ \text { Pow }_{\text {cons }}=\left(\text { cost }_{\text {total }}-\text { cost }_{\text {rest.equip }}\right) / \text { cost }_{\text {generation } / 1 w}\end{array}\right.$

$\tau, \mathbf{k}$, cost $_{\text {generation/1w }}:$ constants.

$\delta_{\text {int }}: \delta_{\text {int }}=\varnothing$, internal transition function, $\lambda: \lambda=\varnothing$, output function and $\mathbf{t}_{\mathbf{a}}: \mathbf{t}_{\mathrm{a}}=\infty$, advancement function of time.

Fig. 7 shows the Refrigerator sub-model modeled on JDEVS environmental.

\section{B.4 Discussion of the simulation results}

After the simulation, the results shown in Fig. 8 were obtained for power consumption.

The Fig. 8 shows the behavior of the Refrigerator for $24 \mathrm{~h}$ in the event of uncontrollable and controllable consumer consumption. In this figure, we can compare the average power consumed by the Refrigerator without reagent that consumed control when the reagent control is started, under the same operating conditions.

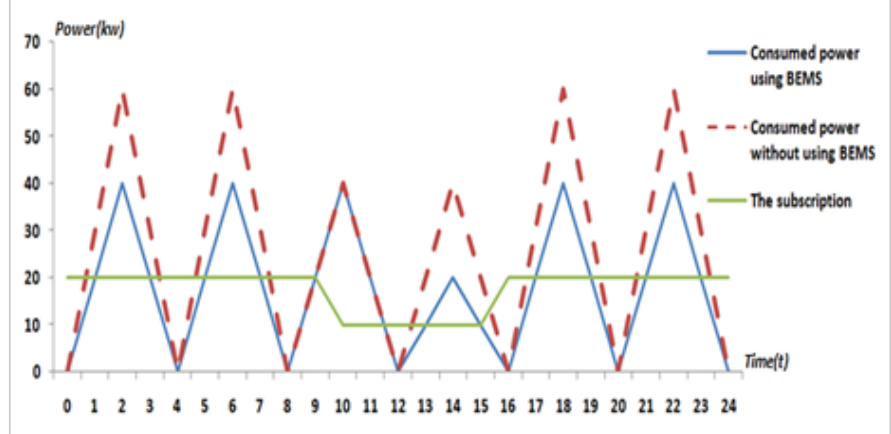

Fig. 8. The consumed power by the Refrigerator

From this curve, we find that the BEMS has not only smoothed the power not to exceed the purchase, but it allowed us to obtain an energy gain $\Delta \mathrm{P}$. The difference in area between the two curves is approximately $17 \%$.
The BEMS receives the power consumption of each type of service (power) but in addition, he receives the instantaneous temperature of the work piece and inside the Refrigerator (permanent services). In return, it returns a temperature and the (ON / OFF) control (enable). It also controls temporary services by sending start time (relative_starting_time) and also (ON / OFF) commands (enable).

Uncontrollable services and services provider energy cannot be controlled.

The anticipative layer is to plan consumption and production of energy when events are planned a few hours or a day in advance.

Anticipated solutions are recalculated when new forecasts are available. The sampling period, denoted $\Delta$, of this layer is of the order of one hour.

The reactive layer helps the anticipative layer to achieve the assignment of the energy resources, taking into account energy constraints and user comfort in real time.

If the energy source is unavailable or restricted, the reactive layer intervenes disabling the use of certain services and balancing consumption and energy production. As against, if there is no unexpected event, this layer occurs not. In this case, its role is to transmit instructions to the local layer.

In Fig. 9, we compared two mechanisms: centralized proposed mechanism and distributed mechanism proposed by S. Abras [15].

We note the quality of the solution obtained is $82 \%$ for the proposed centralized mechanism and $75 \%$ for the distributed mechanism. We also note that the research of the solution takes more time (35\% more) in the distributed mechanism compared with centralized.

We note that the performances of centralized proposed system are better than those obtained with the distributed system. As against, level of robustness in the system based on distributed Multi-Agent Systems, is more robust than centralized system.

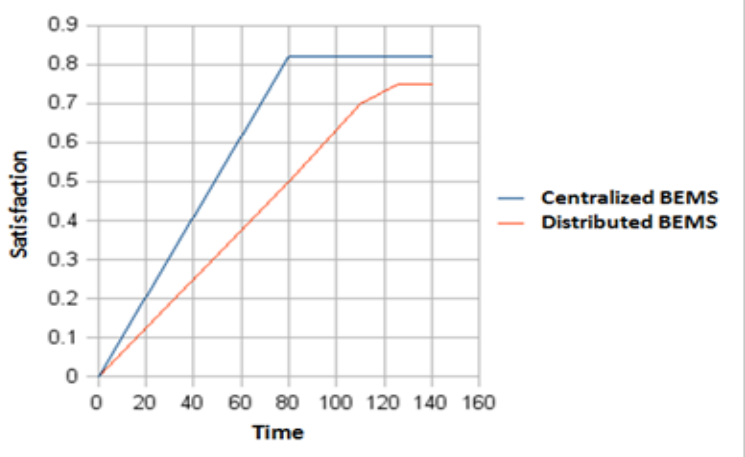

Fig. 9. Satisfaction of solution with centralized and distributed system

\section{CONCLUSION AND FUTURE PROSPECTS}

In this article we presented the modeling and the simulation of a multi-layers BEMS. Our contribution lies on the modeling and the simulation of this system with DEVS for the first time. We have shown that a system of control structured on layers can achieve the desired objective. A proactive layer allows 
calculating in advance the energy assignment plans that take into account the demands of the occupants but also meteorological data and the constraints and needs of energy providers. A reactive layer adjusts plans for assignment to a finer time scale, taking into account unforeseen events. Finally, a local layer allows equipment to follow the guidelines established by the other layers.

We proposed a model that describes the studied system. Based on this model, we have developed an application using the tool of modeling and simulation JDEVS to simulate the behavior of this BEMS. The simulation of the Refrigerator sub model gives good results for the reduction of energy consumption. Our future prospect is the simulation of the behavior of all equipments at the same time in order to test the overall functioning of this BEMS. The result of our work will focus on several points. First, we consider the implementation of this proposal on more complex systems. For this, we want to model the previous BEMS with the formalism DEVS coupled with other tools of Soft Computing to touch all aspects of the real world.

\section{REFERENCES}

[1] K. P. Wacks: Utility load management using home automation, IEEE Trans. on Consumer Electronics, Vol. 37, No.2, pp. 168174, 1991.

[2] K. P. Wacks: The impact of home automation on power electronics, In Proceeding of the Applied Power Electronics Conference and Exposition, pp. 3-9, 1993.

[3] K.Stum, R.Mosier, T. Haasl: "Energy management systems", Technical report, Porland Energy Conservation Inc.(PECI), pp. 41, 1997.

[4] K.Andersen, Klaus: "Modelling the heat dynamics of a building using stochastic differential equations”, Energy and Building, pp. 58, 2000.

[5] Y. Penya, T. Sauter: Communication issues in multi-agent-based plant automation scheduling, Eds. on Intelligent Systems at the Service of the Mankind, W. Elmenreich, J.A.T. Machado, I.K. Rudas,Vol.1, pp. 135-144, Ubooks, Regensburg, Germany, 2004.

[6] D. Hatley, R. Meador, S. Katipamula, M. Brambley: Energy management and control system: Desired capabilities and functionality, Technical report. LtCol. Carl Wouden, USAF, DEAC05-76RL01830.

[7] P. Davidsson, M. Boman: Distributed monitoring and control of officebuildings by embedded agents, Information Sciences, Vol. 171, No. 4, pp. 293-307, 2005.

[8] J. Kämpf and D. Robinson. A simplified thermal model to support analysis of urban resource flows, in Energy \& Buildings, Vol. 39, No. 4, pp. 445-453, 2007.

[9] C. Ménézo, J. Saulnier, D. Lincot, G. Guarracino: Energy, domotics, materials welcome to the home of the future, CNRS international magazine, Vol. 5, pp. 18-27, 2007.

[10]D. Donsez, J. Bourcier, C. Escoffier, P. Lalanda, A. Bottaro: A multi-protocol service-oriented platform for home control applications, $4^{\text {th }}$ IEEE, CCNC'07 : Consumer Communications and Networking Conference, pp. 1174-1175, Jan. 2007.

[11] G. P. Henze, R. H. Dodier: Adaptive optimal control of a grid independent photovoltaic system, Journal of Solar Energy Engineering, Vol. 125, No.1, pp. 34-42, 2003.

[12]D. L. Ha, S. Ploix, E. Zamai, M. Jacomino: Tabu search for the optimization of household energy consumption, In Proceedings of the 2006 IEEE International Conference on Information Reuse and Integration, IRI - 2006: Heuristic Systems Engineering, September 16-18, 2006, Waikoloa, Hawaii, USA, pp. 86-92.

[13]R. R. Negenborn, M. Houwing, B. De Schutter, H. Hellendoorn Adaptive prediction model accuracy in the control of residential energy resources, In: Proc. of the $17^{\text {th }}$ IEEE International
Conference on Control Applications, San Antonio, Texas, pp. 311-316, Sept. 2008

[14]R. Missaoui, H. Joumaa, S. Ploix, S. Bacha, Managing Energy Smart Homes according to Energy Prices: Analysis of a Building Energy Management System, Energy \& Buildings, Vol. 71, pp. 155-167, March 2014.

[15]S. Abras, S. Pesty, S. Ploix, M. Jacomino : Advantages of MAS for the Resolution of a Power Management Problem in Smart Homes, Eds: Yves Demazeau, Frank Dignum, Juan M. Corchado, Javier Bajo, Advances in Practical Applications of Agents and Multiagent Systems, 8th International Conference on Practical Applications of Agents and Multiagent Systems, PAAMS 2010, Salamanca, Spain, 26-28 April 2010,Springer, Advances in Soft Computing, Vol. 70, pp. 269-278.

[16]D. L. Ha, S. Ploix, E; Zamai, M. Jacomino: A home automation system to improve household energy control, 12th IFAC symposium on information control problems in manufacturing, INCOM'2006.

[17]B.P. Zeigler: “Theory of Modelling and Simulation”, Wiley \& Sons, New York, 1976.

[18]L. Capocchi, F. Bernardi, D. Federici, P.-A. Bisgambiglia : BFSDEVS: A general DEVS-based formalism for behavioral fault simulation, Simulation Modelling Practice and Theory, Vol. 14, pp: 945-970, 2006.

[19]R.Missaoui, G.Warkosek, S.Bacha, S.Ploix: Real Time Validation of an Optimization building Energy Management Strategy based on Power-Hardware-in-the-loop Tool, IEEE PES Innovative Smart Grid Technologies (ISGT) 2012, Berlin, Germany.

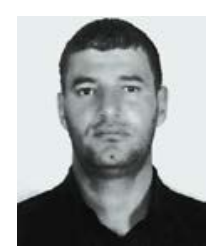

Abdelfettah Maatoug received his engineer diploma in computer science from Institute of computer science, University of Oran, Algeria, in 2000. From 2000 to 2009, he was a specialized engineer of Computer Science and Telecommunication at the high school of Aerial defense of Algiers with research in control of Aerial operation of the air forces of army. He is a $\mathrm{PhD}$ candidate in the National Superior School of Computer Science of Algiers, working on applied research projects in energy management systems for smart building since 2012. He is a member of Computer Science Laboratory of Oran. His recent research focuses on smart cities, smart homes, distributed system, energy management system and multi agents systems.

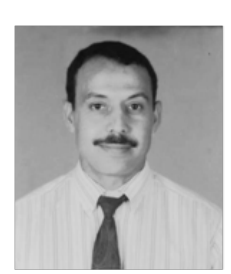

Ghalem Belalem Graduated from Department of computer science, Faculty of exact and applied sciences, University of Oran, Algeria, where he received $\mathrm{PhD}$ degree in computer science in 2007. $\mathrm{He}$ is now a research fellow of management of replicas in data replicas in data grid. His current research interests are distributed system; grid computing, could computing and data grid placement of replicas, consistency, fault tolerance, economic models, energy, Big data, and improved performance in large scale systems and mobile environment. 